Relations industrielles

Industrial Relations

\title{
Lendemain de congrès
} After the Meeting

Volume 1, numéro 7, mars 1946

URI : https://id.erudit.org/iderudit/1023937ar

DOI : https://doi.org/10.7202/1023937ar

Aller au sommaire du numéro

Éditeur(s)

Département des relations industrielles de l’Université Laval

ISSN

0034-379X (imprimé)

1703-8138 (numérique)

Découvrir la revue

Citer cet article

(1946). Lendemain de congrès / After the Meeting. Relations industrielles /

Industrial Relations, 1(7), 1-8. https://doi.org/10.7202/1023937ar

Tous droits réservés @ Département des relations industrielles de l’Université Laval, 1946
Ce document est protégé par la loi sur le droit d'auteur. L’utilisation des services d'Érudit (y compris la reproduction) est assujettie à sa politique d'utilisation que vous pouvez consulter en ligne.

https://apropos.erudit.org/fr/usagers/politique-dutilisation/ 
PUBLIÉ PAR LE DÉPARTEMENT DES RELATIONS INDUSTRIELLES

DE LA faculté des Sciences sociales de laval, Québec

Secrétaire de la rédaction : Jean-Pierre Després

\section{Lendemain de congrès}

Le premier congrès des relations industrielles de la Faculté des Sciences sociales de l'Université Laval a réuni, au Château Frontenac, près de deux cent cinquante personnes. Nos conférenciers invités ont traité leur sujet avec maitrise, mettant particulièrement en relief les aspects pratiques des problèmes qui leur avaient été soumis. Les discussions animées qui suivirent les causeries sont une preuve de l'intérêt que suscita ce congrès. Officiers de syndicats et d'unions ouvrières, directeurs de personnel, contremaîtres, représentants d'associations patronales, directeurs d'entreprises, secrétaires et inspecteurs de comités paritaires, etc., tous ont su apporter aux discussions une objectivité et un brio qui donnèrent à ces délibérations un cachet particulier. Les commentaires et les observations qui nous furent transmis depuis nous permettent d'écrire que ce premier congrès a été un succès. L'expérience a réussi et nous la renouvellerons chaque année: Afin de répondre à la demande générale, le Département des relations industrielles publiera, d'ici quelques semaines, un compte rendu des séances du congrès.

Trois conclusions se dégagent de ces assises.

En premier lieu, ce congrès, le premier du genre organisé dans notre province, a démontré qu'il est très profitable aux employeurs et aux travailleurs de se rencontrer non seulement lorsque survient un conflit, mais aussi pour envisager conjointement tous les problèmes qu'ils ont en commun. Des contacts comme ceux du 11 mars dernier ne peuvent qu'aider à une meilleure compréhension de la solidarité qui existe entre le capital et le travail.

Deuxièmement, ce congrès a prouvé que les travailleurs et les employeurs savent étudier objectivement un problème et en analyser les éléments essentiels. Tous et chacun ont pu se rendre compte que les relations industrielles sont excessivement complexes. L'Etat seul ne

(Suite à la page 8)

\section{After the Meeting}

The first Industrial Relations Conference of the Faculty of Social Sciences of Laval University has gathered, at the Château Frontenac, about two hundred and fifty persons. Our invited speakers discussed their subject with lectureship and particularly threw into relief the practical aspects of the problems submitted to them. The animated discussions that followed every lecture are an evident proof of the interest raised by this Conference. Labour unions officers, personnel managers and directors, foremen, representatives of employers' associations, secretaries and inspectors of parity committees, etc., discussed with objectivity and an interest that coined these talks with character and brio. Comments and remarks since received permit us to state that the Conference has been a successful one. The experiment has been worthwhile ard will be repeated every year from now on. In a few weeks, in answer to a general request, the Industrial Relations Department will publish a verbatim report of this Conference.

Three conclusions emerge from this Industrial Relations Conference.

In the first place, this Conference, the first of its type organized in the Province, has proved very advantageous in giving to Management and Labour the opportunity to gather, not only in case of conflict, but also to mutually discuss all the problems they share together. Contacts like those of March 11th can do nothing else but bring to a better understanding the question of solidarity linking Capital and Labour.

In the second place, this Conference has proved that employees and employers know how to objectively study a problem and to analyse its essential elements. Each and every one have agreed that the problem of industrial relations is one of the greatest complexity. The Government alone cannot find an adequate solution to factual disputes that may arise between Capital and Labour. It is the function of professional organizations (Continued on page 8 ) 


\section{L'ATELIER PRÉFÉRENTIEL}

Une clause d'atelier préférentiel est un engagement d'honneur par lequel l'employeur consent à accorder, dans diverses circonstances particulières, un traitement de faveur, soit au syndicat lui-même, soit à ses membres.

Elle ne consiste pas, comme certains .se l'imaginent parfois, dans la reconnaissance du syndicat par l'employeur comme unique agence de négociation représentant les travailleurs, ce qui s'appelle une clause de reconnaissance syndicale. Et l'on sait, d'autre part, que, depuis l'adoption de la Loi des Relations ouvrières, il n'appartient plus généralement à l'employeur de reconnaitre le syndicat ni de déterminer quels seront les employés que celui-ci représentera.

Il est évident que les clauses de sécurité syndicale que nous ávons déjà étudiées sont, en fait, de la préférence syndicale, mais la clause dite d'atelier préférentiel conserve toute sa signification propre qui la distingue clairement des autres.

Elle comporte, en effet, les particularités suivantes.

D'abord, les non-syndiqués ne sont pas menacés de perdre leur emploi s'ils continuent dans leur détermination de ne point faire partie du syndicat. L'employeur ne force personne à accomplir quoi que ce soit: il se contente de manifester sa sympathie envers le syndicat et de lui accorder sa collaboration en favorisant ses membres à diverses occasions.

De plus, une grande part d'arbitraire est laissée à l'employeur, si bien que, lorsque celui-ci est tout à fait disposé envers le syndicat et applique librement, mais rigoureusement cette clause, celle-ci équivaut, en pratique, à l'atelier syndical le plus strict.

Cette discrimination entre syndiqués et non-syndiqués, tout en demeurant dans les limites permises par la lọi qui défend toute immixtion, de la part d'un employeur, dans les affaires d'un syndicat (1), peut se manifester de mille et une manières. Elle peut avoir pour objet le syndicat lui-même, ou bien les membres du syndicat.

Pour ce qui est du syndicat lui-même, l'employeur peut le favoriser en permettant aux agents d'affaires ou aux chefs de groupe de percevoir les cotisations syndicales sur le lieu du travail, en autorisant l'usage d'un bureau où les chefs du syndicat pourront rencontrer les membres à certaines heures, ou bien, en laissant afficher sur des tableaux dans l'établissement les bulletins annonçant les diverses activités syndicales.

Quant aux membres du syndicat, l'employeur peut leur accorder des faveurs particulières à l'occasion de l'embauchage, des promotions, des mises à pied, des renvois, etc. Si l'employeur, à cause de nouveaux développements dans son entreprise, est obligé d'accrốtre sa main-d'œuvre, il portera une attention spéciale aux candidats qui lui seront présentés par le syndicat. Les membres du syndicat seront aussi les derniers à

(1) 20. Aucun employeur, ni aucune personne agissant pour un employeur ou une association d'employeurs ne cherchera d'aucune manière à dominer ou à entraver la formation ou les activités d'une association de salariés. Ioi des Relations ouvrières, S.R.Q., 1941, chapitre 162A. être mis à pied quand une diminution du personnel sera rendue nécessaire et les premiers à être réembauchés avec la reprise des affaires.

Quoiqu'il n'y ait aucune contrainte d'exercée sur les travailleurs qui ne sont pas syndiqués, on imagine facilement quelles peuvent être, pour le syndicat, les conséquences de l'adoption d'une telle politique par un employeur, car les non-syndiqués se trouvent toujours dans un état d'infériorité vis-à-vis de leurs compagnons de travail membres du syndicat.

Dans les conventions collectives on rencontre plusieurs types de clauses d'atelier préférentiel. Parfois la préférence est limitée à l'embauchage et aux mises à pied; parfois, elle inclut lés promotions et même les droits d'ancienneté, quoique ce dernier cas soit moins connu; parfois, enfin, elle s'étend à toutes les circonstances que nous avons mentionnées. On comprend qu'il soit difficile de rédiger une clause type qui tienne compte de chacun des aspects particuliers à ùne entreprise.

\section{Préférence au syndicat}

"L'employeur facilitera la participation des employés aux activités syndicales légitimes en permettant l'affichage des assemblées et en accordant les congés nécessaires, sans rétribution, aux officiers et membres du syndicat désignés pour négocier une convention de travail, pour assister aux réunions du comité conjoint et aux délibérations des congrès syndicaux."

"L'employeur fournira à chacun des nouveaux employés une copie de cette convention avec une notice attirant leur attention sur le fait que le Syndicat (...) est reconnu comme intermédiaire entre lui et tous les employés et exprimant le désir que ceux de ses employés qui ne sont pas déjà membres du syndicat (...) le deviennent."

"Les jours de paye, l'employeur consent à mettre à la disposition du trésorier du syndicat un bureau adjacent au comptoir du paie-maitre pour la perception des cotisations syndicales."

"L'employeur consent à ce que les cotisations syndicales soient perçues dans son établissement, pourvu que cette perception n'ait point lieu durant les heures de travail ou ne nuise de quelque façon à la production."

"Des tableaux seront fournis par l'employeur pour l'affichage d'avis relatifs aux assemblées, aux cotisations, aux activités syndicales légitimes."

\section{Préférence aux syndiqués}

\section{Embauchage:}

"L'employeur convient de s'adresser au syndicat pour obtenir les travailleurs dont il pourra avoir besoin et s'engage, si possible, à n'employer que des ouvriers qui ont une carte de membre du syndicat (...)

\section{Mise à pied et réemploi:}

"Quand il y aura une réduction temporaire du personnel, les non-syndiqués seront d'abord congédiés, (Suite d la page ?') 


\section{LA SÉCURITÉ INDUSTRIELLE}

Beveridge, dans son bilan des revendications de la nation britannique, préconisait un plan qui avait pour but d'assurer la sécurité de l'individu : la compensation en cas de chômage ; l'aide aux malades.; le soutien des vieillards ; et enfin, la compensation au décès. C'est là une autre preuve que l'humanité désire au moins des palliatifs aux épreuves séculaires auxquelles les hommes ne peuvent se soustraire. En effet, malgré les efforts des économistes, il y a toujours eu des crises de chômage ; la maladie est inévitable ; la vieillesse nous attend tous ...

Il en est autrement de l'accident de travail : dans ce domaine, la compensation cède de plus en plus la place à la prévention. En matière de santé, on mentionne courageusement la médecine préventive comme une réalisation possible et désirable; la sécurité industrielle, pour sa part, est maintenant la résultante de l'application précise de principes scientifiques qui deviennent de plus en plus acceptés partout.

Cet exposé y gagnera en clarté s'il établit clairement notre bilan dans le domaine de la sécurité industrielle ; il sera alors plus facile de tirer des conclusions et de songer aux moyens pratiques d'améliorer les méthodes de prévention, de mieux grouper les bonnes volontés et d'obtenir, en fait, que l'usine devienne un endroit ou l'ouvrier gagne sa vie en toute sécurité.

\section{LES REALISATIONS EN MATILHE DE StGURITt} DANS LE QUEBEC

La première législation de prévention d'accidents adoptée en Amérique du Nord, date de 1867 : la Législature du Massachusetts, en l'année même de la naissance de la Confédération canadienne, constituait un Service d'inspection du Travail. Dix ans plus tard, les employeurs de cet Etat étaient assujettis à l'obligation légale de poser des gardes adéquates sur les machines dangereuses. Le seul autre Etat américain avant le Québec à légiférer indirectement dans ce domaine fut l'Alabama qui, avec le Massachusetts, adoptait, en 1885, une législation imposant à l'employeur la responsabilité en cas d'accident de travail : nous y retrouvons les principes de nos lois actuelles de compensation d'accidents.

Le Québec, se conformant comme l'Ontario aux recommandations d'une commission parlementaire fédérale, adoptait en 1885 l'Acte des Manufactures qui est devenu après plusieurs refontes notre Loi des établissements industriels et commercioux ; c'est la loi organique de sécurité et de prévention des accidents à l'usine. Son champ d'application est très vaste et le personnel d'inspection, nombreux et de plus en plus expérimenté, peut suffire à la mission qui lui a été confiée. On peut donc conclure que la province de Québec, dans le domaine législatif de la prévention des accidents, n'a pas été retardataire.

Le patronat et la classe ouvrière ont cependant vite réalisé qu'ils avaient aussi des responsabilités : les em- ployeurs, en raison d'un désir de diminuer des accidents provenant du fonctionnement de leur industrie et aussi pour éliminer une source de perte d'argent et d'ênergie; les ouvriers, afin de cesser de vivre au travail dans la crainte de l'accident et de ses conséquences désastreuses. La première intervention devait se manifester par la formation de sociétés patronales de prévention d'accidents; plus tard, elle prit la forme d'un effort conjoint des employeurs et des employés pour la prévention des accidents ; dans le Québec, on suivit l'exemple tracé aux Etats-Unis. Tout comme en 1915, un groupe d'enthousiastes jetait les bases du National Safety Council américain à la suite de l'adoption d'une législation portant sur la réparation des accidents du travail, de même, dans le Québec, la mise en vigueur de notre Loi des accidents du Travail, il y a seize ans, a précédé de quelque temps à peine la constitution d'une Association patronale de prévention des accidents du Travail. Ce groupement, aidé financièrement par la Commission des accidents du Travail, est de plus en plus actif. Il a de belles réalisations à son crédit et collabore intimement avec le Service gouvernemental d'inspection des établissements industriels et commerciaux. Son domaine comprend surlout l'éducation et la propagande.

Par ailleurs, nombreuses sont les usines où fonctionnent des comités mixtes de prévention d'accidents. Les ouvriers participent aux campagnes d'éducation, contribuent à déceler les risques et procèdent aux recommandations appropriées ; et l'expérience a prouvé que ces efforts sont couronnés de succès.

Tout comme dans un plan général des relations industrielles, nous retrouvons ici la collaboration tripartite :

$1^{\circ}$ celle de l'employeur qui, en plus de ses sentiments humanitaires, en arrive à son but légitime de réduire le coût de sa production au strict minimum en évitant des contributions excessives et inutiles au fonds commun de réparation d'accidents;

$\mathscr{2}^{\circ}$ celle de l'employé, soucieux de sécurité, très au courant soit directement soit indirectement des conséquences del'accident de travail et pour lui-même et pour ses dépendants ;

$3^{\circ}$ celle de l'Etat qui a, pour le bien commun, la mission, en élaborant et en appliquant des minima de sécurité, de suppléer à la carence de certains employeurs et de forcer les récalcitrants à suivre un mouvement essentiellement social et nécessaire.

Tout comme dans le domaine des relations industrielles, le deuxième conflit mondial nous a donné une profonde leçon en matière de sécurité industrielle. La nécessité en Amérique du Nord d'un effort total de guerre, exigeant une production sans cesse accrue et requérant l'intervention des gouvernements pour la prévention des arrêts de travail, a aussi intensifié le désir d'éliminer l'arrêt individuel de travail provenant de l'accident. Cette leçon va continuer de porter ses fruits et nous pourrons apprécier immédiatement ses conséquences. 


\section{LES RELATIONS INDUSTRIELLES DANS L'ÉTAT MODERNE}

En allant jusqu'au fond de mon analyse, je peux trouver la cause profonde des frictions, conflits, grèves et lockouts qui continuent à éclater périodiquement dans l'état d'instabilité qui marque notre régime économique. Dans la période d'entre les deux guerres, celuici était frappé, par intervalles plus ou moins réguliers, de crises caractérisées par la mévente, la baisse des prix et des salaires, le chômage. Pendant la guerre et sous l'impulsion vigoureuse des gouvernements, le potentiel des économies nationales a été développé à son maximum. Toute la main-d'œuvre a été drainée vers les industries de guerre; le chômage a disparu complètement; les salaires ont augmenté.

Maintenant, nous sommes entrés dans la phase de reconversion. Du fait de la suppression des heures supplémentaires, le salaire hebdomadaire a une tendance à diminuer et le spectre du chômage recommence à hanter l'esprit des ouvriers. Après la période dé guerre, au cours de laquelle les conditions de travail étaient fixées à un niveau relativement élevé à l'aide d'une réglementation rigoureuse, les ouvriers voient de nouveau poindre à l'horizon l'insécurité et la possibilité de l'avilissement de leurs conditions.

Et alors que la procédure conventionnelle semble suffire pour les périodes d'évolution plus ou moins normale, elle paraît nettement insuffisante dans les moments de grandes secousses qui ébranlent périodiquement notre régime économique.

Justice et liberté restent toujours les aspirations profondes des masses travailleuses, mais les revendications qu'inspirent ces aspirations évoluent avec le temps. Il y a un demi-siècle, lorsque les ouvriers durent œuvrer pendant de longues heures pour une maigre pitance, ils revendiquaient principalement un accroissement de salaires et une réduction de la durée du travail. A présent, les revendications ouvrières portent surtout sur la garantie d'un emploi continu et d'un revenu stable.

Pour ceux que frappent les insécurités de toutes sortes qui caractérisent notre époque, pour ceux qui sont sensibles aux affres des sans-travail ayant à charge des familles, il n'y a rien de déraisonnable dans ces revendications.

Je m'adresse aux patrons qui sont déjà allés si loin dans la voie de la compréhension sociale à l'égard de

(1) Nous reproduisons, ici, la conclusion de la magistrale conférence prononcée, au Congrès des Relations industrielles de Laval, par M. JEF RENs, sous-directeur du Bureau international du Travail. Le texte complet de l'exposé de M. Rens, intitulé Les relations industrielles dane l'Etat moderne, paraîtra dans le compte rendu du Congrès qui sera imprimé sous peu. leurs travailleurs et leur dis: si vous réalisez toute la portée de ces revendications ouvrières, si vous montrez de la sollicitude pour ce désir de vos travailleurs d'échapper avec les leurs à cette étreinte débilitante des cycles économiques, si vous avez la ferme volonté d'aboutir, par vos efforts conjugués, à une production de paix continue, capable d'occuper tous les bras disponibles et de répondre largement aux besoins de consommation des masses travailleuses, vous aurez apporté votre contribution à la solution du problème des relations industrielles.

Aux travailleurs je dis : continuez à vous inspirer des grands idéaux de justice et de liberté qui ont été les principaux moteurs de votre ascension. Continuez à rallier autour de ces idées autant de vos compagnons de travail que possible. Efforcez-vous constamment de conformer vos revendications à ces idées. En formulant votre programme, soyez justes et raisonnables. Précisez vos conceptions du progrès social et continuez à les propager parmi vos concitoyens. Essayez d'impressionner favorablement l'opinion publique par la discipline de vos organisations et de votre action, ainsi que par la justice de votre cause. Ayez le souci constant d'apparaitre comme les promoteurs d'une meilleure forme de la société, d'une forme plus élevée de civilisation.

Aux représentants de l'Etat, aux Gouvernements, je me permets d'adresser les conseils que voici: à vous incombe la tâche la plus dure. Il vous appartient de veiller à ce que l'activité économique assure effectivement la plénitude de l'emploi et réponde aussi largement que possible aux besoins de la masse des citoyens en général, des couches laborieuses en particulier, tout en sauvegardant les libertés individuelles essentielles. Ceci nous ramène au dilemme auquel j'ai fait allusion. La solution de ce dilemme ne me paraît pas une tâche surhumaine. C'est d'elle que dépend, à mon humble avis, le développement harmonieux des relations industrielles. En effet, la collaboration, la collaboration confiante, la collaboration agissante, entre employeurs et travailleurs se substituera à l'antagonisme entre ces deux catégories dans la mesure précise où la légitimité du progrès social sera admise et où les employeurs et le gouvernement assureront aux ouvriers la pleine utilisation de leur travail et l'amélioration graduelle de leurs standards de vie.

Formulons tous ensemble le vœu ardent que chacun comprenne sa tâche dans cette œuvre commune qui consiste à délivrer les nations de la misère et de l'insécurité et à permettre ainsi leur évolution dans la paix entre tous les groupes de producteurs. 


\section{INDUSTTRIAL SAFETY}

Beveridge, in his recapitulation of the needs of the British Nation, outlined a plan having for its object the safety of the individual : Unemployment Insurance ; Sick Benefits ; Old Age Pension and finally Life Insurance. This was a further proof that humanity strives, at least, for palliatives to secular unhappiness, the memory of which cannot be taken away. In spite of the efforts of the best economists, there have always been mass unemployment periods; sickness cannot be avoided; old age is also unavoidable.

We have another story inasmuch as the industrial accident is concerned; in this field, compensation gradually dissappears to be replaced by prevention. In the domain of health, some mention, with courage, preventive medecine as a possible and desirable achievement. Industrial safety has already become the result of the precise enforcement of scientific principles which are more and more accepted everywhere.

Better clarification will be obtained if we summarize our achievements in the fields of industrial safety. It will then be easier to draw conclusions and to arrive at practical means to give better efficiency to preventive methods, to group more citizens of good will and to make out of the industrial plant a place where the workman earns his living safely.

\section{The Quebec Achievements IN The FIELd of SAFETy}

The first legislation dealing with accident prevention in North America dates back to 1867 ; the State of Massachusetts' Legislative Assembly in the very years of the coming into being of the Canadian Confederation, formed a Labour Inspection Service. Ten years later, the employers of that State became legally bound to guard effectively dangerous machineries. The only other government in North America before Quebec to legislate indirectly in this field was the Alabama State authority which, together with Massachusetts, adopted in 1885 a legislation imposing upon management some responsibilities in case of accident ; we still find in such legislative measures the principles of our present Workmen's Compensation Acts.

The Quebec Government, together with that of Ontario, enforced the recommendations of a Federal Parliamentary Commission and adopted in 1885, the Manufacturing Establishments Act which became, after many revisions, our present Industrial and Commercial Establishments Act : it is our basic legislation dealing with safety and accident prevention in manufacturing concerns. Its scope is very broad and the inspection staff, numerous and ever gaining in experience, lives up to its tasks. It is, consequently, to be concluded that the Province of Quebec, in the field of accident prevention legislation, has not been backward.
Management and Labour have quickly realized that they also have responsibilities ; employers, on account of a well founded desire to cut down accidents derived from the functioning of their industry and also to eliminate a loss of money and energy ; workers, in order to stop living in manufacturing establishments in the fear of industrial accidents and of their disastrous consequences. The first practical step to fulfill that desire took the shape of the constitution of Accident Prevention Associations of Employers, and later on, was followed by a common effort of Management and Labour in the field of accident prevention ; in Quebec, the example set down in United States was followed. In 1915 a group of enthusiastic citizens went ahead with the constitution of the American National Safety Council, following the adoption of legislation dealing with Workmen's Compensation; in Quebec, the enforcement of our Workmen's Compensation Act, sixteen years ago, was almost immediately followed by the constitution of the Quebec Industrial Accidents Prevention Association. This group benefitting by financial aid from the Workmen's Compensation Board is more and more active. Its scope of activities comprises namely Education and Propaganda.

Various surveys have shown that in many plants, Joint Prevention Accident Committees are operating. Workers partake in educational drives, are on the lookout for accident risks and make appropriate recommendations ; experience has definitely proved that such efforts are successful.

Just as on the general level of industrial relations, here again we find a tripartite cooperation :

$1^{\circ}$ From management which aside from human reasons, is anxious to reach its legitimate objective to cut down production cost to a strict minimum by a voiding excessive and useless levies to the common accident compensation funds ;

$2^{\circ}$ From labour desirous of safety and untortunately too well aware, directly or indirectly, of the consequences of an industrial accident for the worker and his dependents ;

$3^{\circ}$ From the State, which, for the common good, has for its duties to draft and enforce safety standards, to act on behalf of a group of employers not safety minded and to compel objectors to follow an essentially social and necessary movement.

Just as in the field of industrial relations, the second world-wide struggle has given us a lesson in the scope of industrial safety. The necessity in North America for a total war effort, which necessitated an always enlarging production and requiring State intervention to do away with stoppages of work, has also increased the desire to eliminate the individual stoppage of work following an accident. Such lesson will achieve its purpose and we will soon be able to appreciate its consequences. 


\section{L'UNIVERSITÉ ET LES RELATIONS INDUSTRIELLES}

Sera-t-il dit que devant un appel aussi pressant l'Université va rester insensible et se reconnaître impuissante? Non, car s'il est vrai que les Relations industrielles ont besoin de l'Université, il ne l'est pas moins que l'Université, de son côté, peut répondre à ce besoin.

Celle que l'on a appelée " école de haut savoir et source de directives sociales ", on la nomme aussi le cerveau de la nation. Pour répondre adéquatement aux besoins de celle-ci, ce cerveau possedde en aussi grand nombre qu'il en faut des Facultés. Par exemple, la Faculté de médecine pour veiller sur la santé physique du corps social; la Faculté de droit pour établir les cadres juridiques de la vie nationale et assurer le règne de la justice entre les citoyens ; la Faculté de philosophie qui recherche et diffuse les lumières de la sagesse ; la Faculté de théologie pour subvenir aux besoins spirituels les plus élevés; la Faculté des sciences qui cherche -à découvrir les lois de la nature pour mettre celle-ci plus entièrement et plus efficacement au service de l'homme.

Si donc l'Université a raison de s'occuper de la vie religieuse, intellectuelle, juridique, physique et matérielle de la nation, n'est-elle pas aussi justifiée de veiller sur sa vie sociale, toute faite de relations : d'abord entre les individus, puis entre ces derniers et les sociétés et enfin entre les sociétés elles-mêmes ? Voilà bien pourquoi elle a sa Faculté des sciences sociales, qui forme elle aussi des "professionnels", dont la tâche propre est de chercher à créer ou à maintenir dans la société de bonnes relations. Et puisque parmi les relations sociales, les relations industrielles présentent des difficultés spéciales, il était normal que cette Faculté mît sur pied un Département des relations industrielles pour travailler à résoudre le mieux possible ces difficultés.

Mais alors, plus précisément, de quelle façon l'Université peut-elle contribuer à de meilleures relations industrielles?

D'abord, par ses travaux de recherche. Lorsqu'on se propose, en effet, d'améliorer un objet quelconque, la première chose à faire est de le bien connaître, de l'étudier positivement, d'en saisir de façon bien réaliste la nature et les diverses propriétés.

Or, est-il institution mieux placée que l'Université pour étudier efficacement et dans toute leur ampleur, les réalités sociales? Elle a pour cela toutes les connaissances voulues ayant à sa portée les secours de la philosophie, de la sociologie, de la doctrine sociale de l'Eglise, comme aussi de toutes les sciences connexes

(1) Voici le texte de la seconde partie de l'allocution prononcée par le T. R. P. Georges-Henri Ltevesque, o.P., doyen de la Faculté des Sciences sociales de Laval, à l'ouverture du Congrès des relations industrielles au Château Frontenac. Le texte intégral da cette allocution paraîtra dans le compte-rendu du Congrès qui sera imprimé sous peu, qu'elle peut utiliser à son gré. Elle a de plus toutes les techniques scientifiques qui conviennent. Ses facilités d'observation sont immenses, elle peut faire de vastes enquêtes sociales, s'appuyer sur des statistiques scientifiquement compilées et interprétées. De plus, et cela est bien précieux, c'est elle qui offre les meilleures garanties d'impartialité, puisqu'elle jouit de toute l'indépendance et de l'éloignement nécessaires à une juste appréciation des hommes et des choses. Située sur un sommet, elle a une meilleure vue sur les ensembles et ses perspectives sont d'autant plus réalistes qu'elle voit les problèmes particuliers en fonction de l'ordre général. Mais si haut qu'elle plonge son règard, elle n'est cependant pas pour autant isolée du réel concret, comme on peut être quelquefois enclin à le lui reprocher. Au contraire, elle s'efforce de maintenir un contact très étroit et de garder des relations constantes avec les entreprises et la vie industrielle elle-même; elle s'applique aussi à se tenir très près des groupes ouvriers ou patronaux, à l'aide desquels, d'ailleurs, elle aime à conduire ses plus fructueuses expériènces sociales. Le seul fait du présent congrès n'en est-il pas un magnifique exemple? Et s'il en fallait un autre, je le trouverais facilement dans ceci que nos professeurs se trouvent à peu près tous engagés de quelque façon dans la vie sociale industrielle par les travaux qu'ils accomplissent, soit pour les patrons, soit pour les unions ouvrières, soit pour l'Etat. C'est donc dire que par ses travaux de recherches, l'Université peut rendre de très grands services aux relations industrielles. C'est là ce que j'appellerais volontiers le moment sociologique de son action.

Il en est un autre, cependant, plus proprement social, celui-là, et certes non moins important : celui de la formation de spécialistes en relations industrielles. L'Université prépare tout d'abord des agents de relations industrielles, à qui elle donne toute la formation doctrinale, sociologique, technique, psychologique et morale voulue pour qu'ils deviennent de véritables instruments de coopération, d'harmonie et de paix sociales et dont l'action s'inspirera non pas des intérêts de tel ou tel groupe (patrons ou ouvriers), mais du bien commun de toute la société. Car, ne l'oublions jamais, il y a, dans tout conflit industriel, un tiers intéressé autant qu'invisible mais plus important peut-être que les deux autres et dont la présence réelle doit se faire sentir dans les conseils industriels: c'est la société, le peuple, la masse anonyme des consommateurs dont nous sommes tous et sur le dos de qui se règlent malheureusement trop souvent maintes querelles sociales. Il ne faut pas perdre de vue que les professions et les métiers existent pour faire vivre leur homme, sans doute, mais aussi et beaucoup pour faire vivre les hommes en société. C'est là l'intention formelle et bien explicite de la grande loi naturelle de la division du travail. Et c'est précisément pour cela que la question des rapports entre le capital et le travail s'appelle partout, et avec un sens 
très profond, la question sociale : la société y est aussi directement intéressée que le capital et le travail.

Mais l'Université ne se borne pas à former des agents de collaboration, elle forme aussi des chefs pour les organisations ouvrières. Par son enseignement proprement universitaire d'abord, et ensuite par le truchement de son Service extérieur d'éducation sociale, elle s'efforce de préparer, pour les syndicats ouvriers, des hommes véritablement aptes à défendre, et le mieux possible, les intérêts bien compris et les droits inaliénables de la personne ouvrière. De vrais chefs qui seront non seulement les porte-parole éclairés des justes revendications de la classe ouvrière, mais aussi, les éducateurs sociaux qui donneront aux travailleurs le souci de leur grande dignité, tout en leur apprenant à maintenir leurs exigences dans les normes de la justice, à juger de leurs problèmes en fonction du bien commun et à maintenir sous le contrôle de la prudence, sans pour autant les paralyser, leur dynamisme spontané et leur naturelle tendance à la revendication.

L'Université prépare enfin, pour les patrons, les experts dont ils ont besoin. Ces hommes apprendront à l'Université que si les patrons ont des devoirs ils ont aussi des droits, que s'ils ont des droits ils ont aussi des devoirs; que la propriété est un droit naturel, mais qu'elle a aussi ses charges sociales; que les ouvriers ne sont pas des machines et que leur travail n'est pas une vulgaire marchandise ; que le travailleur est une personne humaine, et que, de ce fait, les conditions de son travail ne doivent pas être celles des esclaves ; mais des hommes libres, égaux en dignité de nature, créés à l'image de Dieu et rachetés par son sang.

Voilà, mesdames et messieurs, trop brièvement rappelé, le rôle de l'Université dans le domaine des relations industrielles. Avons-nous assez souligné, cependant, le caractère essentiellement spirituel et moral de ces relations? C'est là un fait qui rend encore plus nobles et plus pressantes les tâches que nous venons d'assigner à l'Université ; et c'est sur cette dernière considération que je veux achever mon discours. Les temps mémorables que nous vivons sont témoins d'un retour offensif du matérialisme révolutionnaire. On dirait que les hommes ont perdu de vue les fins de la vie humaine pour se rabattre sur la seule contemplation des moyens. L'intelligence humaine dont les lumières ont pu percer les secrets les plus cachés du monde physique semble bien pauvrement outillée pour assurer à l'humanité un progrès social et moral comparable à ses progrès matériels. Serait-ce vraiment que la matière est la seule réalité qui existe et au-delà de laquelle il n'y a rien que superstitions religieuses ou autres? Bien des faux docteurs le prétendent aujourd'hui, et avec d'autant plus de chance d'être crus que les forces spirituelles semblent accuser un scandaleux recul. $\mathrm{Si}$ l'homme contemporain ne retrouve pas bientôt son âme, il est à craindre qu'il ne périsse sous les décombres d'une civilisation que les plus belles puissances intellectuelles et morales de ses ancêtres ont mis des siècles à construire, et que son incompréhensible égarement menace de laisser détruire en un instant.

Si le siècle qui a vu, comme pas un autre, le fléau des crises économiques et des grands conflits industriels, à qui fut donné le spectacle des deux plus horribles guer- res qui aient jamais ensanglanté la terre des hommes, qui voit présentement se dresser au lendemain même de l'hécatombe, dominant celui de la famine, le spectre plus épouvantable encore d'une troisième guerre mondiale, si ce siècle, dis-je, ne revient à la foi en l'esprit, il faudra désespérer de la destinée humaine. Ah ! si les hommes mettaient autant d'amour à développer leurs (nergies spirituelles pour le plus grand bien de toute la société qu'ils mettent d'intelligence à harnacher celles de la matière pour la destruction de leurs frères, comme le monde serait plus heureux. Si au lieu des chəmps de bataille, où l'on se tue, il n'y avait que des champs de travail où, loin de se combattre, l'on peinerait ensemble les uns pour les autres ; si les humains savaient retrouver dans leur détresse même les raisons de leur fraternité perdue ; s'ils voulaient croire un instant aux promesses de la justice, et s'ils pouvaient surtout, oui s'ils pouvaient jamais communier aux douceurs bienfaisantes de la charité chrétienne, comme la vie serait plus belle partout, dans les chantiers de l'industrie comme sur la scène du monde! Il est bien malheureux que nous soyons si loin de tout cela, mais rien n'est perdu tant que des hommes restent capables de se rencontrer sérieusement, comme vous le faites en ce moment, tant que vacille encore au fond troublé des consciences humaines la moindre lueur de foi en des jours meilleurs. Ce n'est certes pas nous, chrétiens, qui croyons à l'existence de'la Providence et à la bonté d'un Père qui n'abandonne jamais ses enfants, ce n'est pas nous qui manquerons d'espérance.

Il faut croire au contraire que dans l'abîme profond de son malheur, à la terrible lumière des catastrophes qui ont précipité sa chute, l'homme saura reprendre la main blessée de son frère et sans regarder les ruines, oubliant dans un grand pardon toutes les haines du passé, marcher avec lui sur le sentier si longtemps désert mais désormais retrouvé de la paix, cette tranquillité de l'ordre.

\section{L'ATELIER PRÉFÉRENTIEL}

(Suite de la page \&)

puis les syndiqués, en tenant compte de leur expérience et de leur droit d'ancienneté."

“ L'employeur convient de n'embaucher aucun employé tant qu'un ou plusieurs des membres du syndicat, déjà mis à pied par l'employeur pour cause de diminution de travail, n'ont pas repris celui-ci. Si ce ou ces membres du syndicat, après avoir reçu un avis, négligent de reprendre leur travail dans les 48 heures, l'employeur pourra embaucher les candidats qu'il désirera."

\section{Promotions:}

"Lorsqu'une position deviendra vacante, une préférence sera accordée aux ouvriers membres en règle avec le Syndicat, mais en tenant compte de leur expérience, de leur compétence, ainsi que de leur droit d'ancienneté."

Gerard Dion. 


\section{BIBLIOGRAPHIE}

Cette liste, forcément incomplete, indique, sans approuver pour autant leur contenu, certaines publications récentes ou le lecteur pourra se renseigner sur l'évolution des idées et des faits dans le domaine des relations industrielles.

\section{VOLUMES ET TRACTS}

Rencontre de deux mondes - La crise d'industrialisation du Canada français, Hughes, Everett C., Parizeau, Montréal (traduit de l'anglais par Jean-Charles Falardeau).

Manual of Employment Interviewing - Research Report No. 9 American Management Association, New-York.

Counselling Methods for Persornel Workers, Gargetr, Annett Family Welfare Association of America, New-York, 1945.

Conference Leadership Training, M ACLIN, Edward S. et McHenRY, Paul T. - Deep River, Conn. - National Foremen's Institute, 1945.

The Worker and the Law, M ARqulres, H. D. E. - Workmen Benefit Fund.

Rapport général du Ministère du Travail de la province de Québec - pour l'année finissant le 31 mars 1945, Québec.

Salaires et heures de travail au Canada - 1943. Supplément à la Gazette du Travail, juin 1945, Ottawa.

The International Labour Movement, Price, John-Oxford University Press, 1945, Toronto.

Comment sauvegarder l'entreprise privée - Premier Congrès Patronal - Association Professionnelle des Industriels, 1916.

Trends in Collective Bargaining - Summary of Recent Experience, WiLliamson S. T., et HARRIs, Herbert - Twentieth Century Fund, 1945, New-York.

\section{PÉriodigues \\ CANADA}

The Rand Formula for Union Security - The Canadian Unionist, vol. XX, n॰2, février 1946, Ottawa.

All Ford Employees Must Pay Dues - The Canadian Unionist, vol. XX, $\mathrm{n}^{\circ} 2$, février 1946, Ottawa.

Union-Management Co-operation, Corbett, Jos. - Trades and Labor Congress Journal, vol. XXV, no 2, février 1946, Ottawa.

Une campagne d'organisation coordionnée, solution à nos problèmes - Trades and Labor Congress Journal, vol. XXV, $\mathrm{n}^{\circ} 2$, février 1946, Ottawa.

HIgh Income Means More Than High Wage Rates, SeLry, Richard - The Labour Review, février 1946, Ottawa.

Mesures recommandées par la Commission canadienne de la jeunesse au sujet de l'emploi des jeunes - La Gazette du Travail, janvier 1946, Ottawa.

Administration des allocations familiales au Canada - La Gazette du Travail, janvier 1946, Ottawa.

Róunion à Paris de la Fódération syndicale mondiale - La Gazette du Travail, janvier 1946, Ottawa.

Les méthodes de consultation des employeurs et des travailleurs appliquées pendant la guerre aux États-Unis et en Grande-Bretagne - Revue internationale du Travail, octobre 1945, Montréal.

Participation des organisations professionnelles à l'administration des industries nationalisées en France - Revue internationale du Travail, octobre 1945, Montréal.

G6nie social - Travail, mars 1946, Montréal.

Les droits de l'union minori taire - Le Travail, mars 1946, Montréal.

\section{ETATS-UNIS}

Are Management's Views of Supervision Faulty?, File, QUentin W. -- Personnel Journal, janvier 1946, volume 24, n० 7, NewYork.

Representation in Management - The Government of France Personnel Journal, janvier 1946, vol. 24, n 7, New-York.

Personnel Work on the Grill, Stewart, D. A., et Bolano:ich, D. J. - Personnel Journal, février 1946, volume 24, n8, NewYork.

\section{LENDEMAIN DE CONGRÈS}

\author{
(Suite de la page 1)
}

peut apporter une solution adéquate aux conflits d'intérêts qui peuvent surgir entre le capital et le travail. Il appartient aux associations professionnelles de prendre leurs responsabilités et d'étudier les aspects techniques des relations industrielles. C'est pourquoi ces associations doivent consacrer plus que jamais leur attention aux recherches et aux analyses qui s'imposent.

Enfin, ce congres a permis aux associations patronales et ouvrières de mieux évaluer la contribution que peut apporter l'Université au développement harmonieux des relations industrielles. L'allocution du T.R.P. G.-H. LEvESQUE, o.P., dont nous reproduisons un extrait dans ce Bulletin, laisse entrevoir des possibilités que naguère l'on soupçonnait à peine. Au cours du congrès, notre Département a établi des contacts que nous savons apprécier à leur juste valeur. En toutes circonstances, les employeurs et les travailleurs peuvent être assurés de notre coopération la plus entière.

\section{AFTER THE MEETING}

(From page 1)

to assume their responsibilities and study the technical aspects of industrial relations. It is the reason why these organizations must, more than ever, turn their attention to the research and analysis work taking paramount importance.

Finally, this Conference permitted to employer and employee organizations to get better acquainted with the contribution the University can provide towards the harmonious development of industrial relations. The introduction lecture by R.F. G.-H. Lévesque, o.P., a passage of which is reprinted in this Bulletin, drops a hint of the possibilities the existence of which we were not even suspecting a short time ago. During the Conference our Department has set up connections, the value of which we highly appreciate. In all circumstances, employers and employees may rest assured of our most entire co-operation.

Warm or Cold Management?, Loken, Robert D. - Personnel Journal, février 1946, volume 24, ${ }^{\circ} 8$, New-York.

A Great Union Comes Home-American Federationist, février 1946, Washington.

Strikes and the Public Interest, Slichter, Sumner H. - The Yale Review, p. 207.

What Management is Asking Unions For, Rice, James O. $-\mathrm{Na}$ tion's Business, janvier 1946.

Educational Programs for Employees - The Dartnell Corporation, Chicago. Experience Sheet, 1945.

Wage Policy and Full Employment, Braunthal, Alfred - International Postuar Problems, vol. IIl, $\mathrm{n}^{\circ} 1$, janvier 1946, New-York.

Foremen's Association of America - Monthly Labor Review, février 1946, United States Department of Labor, Washington.

Housing Conditions in Canada - Monthl $l_{;}$Labor Review, février 1946, United States Department of Labor, Washington.

What Labor Expects of Employment Service, Soderstrom, R. G. - American Federationist, mars 1946 , vol. $53, n^{\circ} 3$, Washingion. 